\title{
A Wireless Sensor Networks-based Intelligent System for Library Air Quality Monitoring
}

\author{
https://doi.org/10.3991/ijoe.v12i11.6227 \\ Zhang Xiao-guang ${ }^{1}$, Zhao Yijie ${ }^{1}$, Zhao Wei ${ }^{1}$, Xu Wei-wei ${ }^{2}$, Ji Wen-chao ${ }^{1}$ \\ ${ }^{1}$ Hebei North University, Hebei, China \\ ${ }^{2}$ Zhangjiakou Electronic Education, Hebei, China
}

\begin{abstract}
A system for monitoring library air quality by using the emerging technology of Wireless Sensor Networks (WSN) was proposed. Wireless sensor networks can sense and collect data, as well as carry out a real-time monitoring of various environmental parameters, and send data to the end user. Simulation results prove that the proposed system is a power-saving, low-cost and easy-to-use monitoring system, which is efficient enough to be used as a reliable library air quality (LAQ) monitor.
\end{abstract}

Index Terms-collecting data, library air quality, monitor system, wireless sensor networks

\section{INTRODUCTION}

The rapid advances in electromechanical (MEMS) sensor technology, growing popularity of wireless networks and continuing development of embedded computing devices have led to the emergence of wireless sensor networks (WSN). It has raised considerable interest in the research community as it has great potential to revolutionize many science and engineering domains. A wireless sensor network (WSN) is built of "nodes"-from a few to several hundreds or even thousands, where each node is connected to one (or sometimes several) sensors. Each such sensor node has typically several parts: a radio transceiver with an internal antenna or connection to an external antenna, a microcontroller, an electronic circuit for interfacing with the sensors and an energy source, usually a battery or an embedded form of energy harvesting. A sensor node might vary in size from that of a shoebox down to the size of a grain of dust, although functioning "motes" of genuine microscopic dimensions have yet to be created [1-2].

The development of wireless sensor networks was motivated by military applications such as battlefield surveillance; today such networks are used in many industrial and consumer applications, such as industrial process monitoring and control, machine health monitoring, and so on [3]. In recent years, WSN are being developed for a wide variety of applications including Internet of Things, environmental monitoring, home automation, remote healthcare and patient monitoring, industrial sensing and diagnostics, asset tracking and supply chain management [4].

In a typical WSN platform, there are three sub architectures composing the technology. These are the hardware, software, and network architectures, as seen in figure 1. Although the three sub-architectures are tightly coupled in a given WSN system, the sub-architecture can be considered a separate discipline or an independent body of knowledge.

The hardware architecture focuses mainly on the hardware design of the source nodes, which make up the ma-

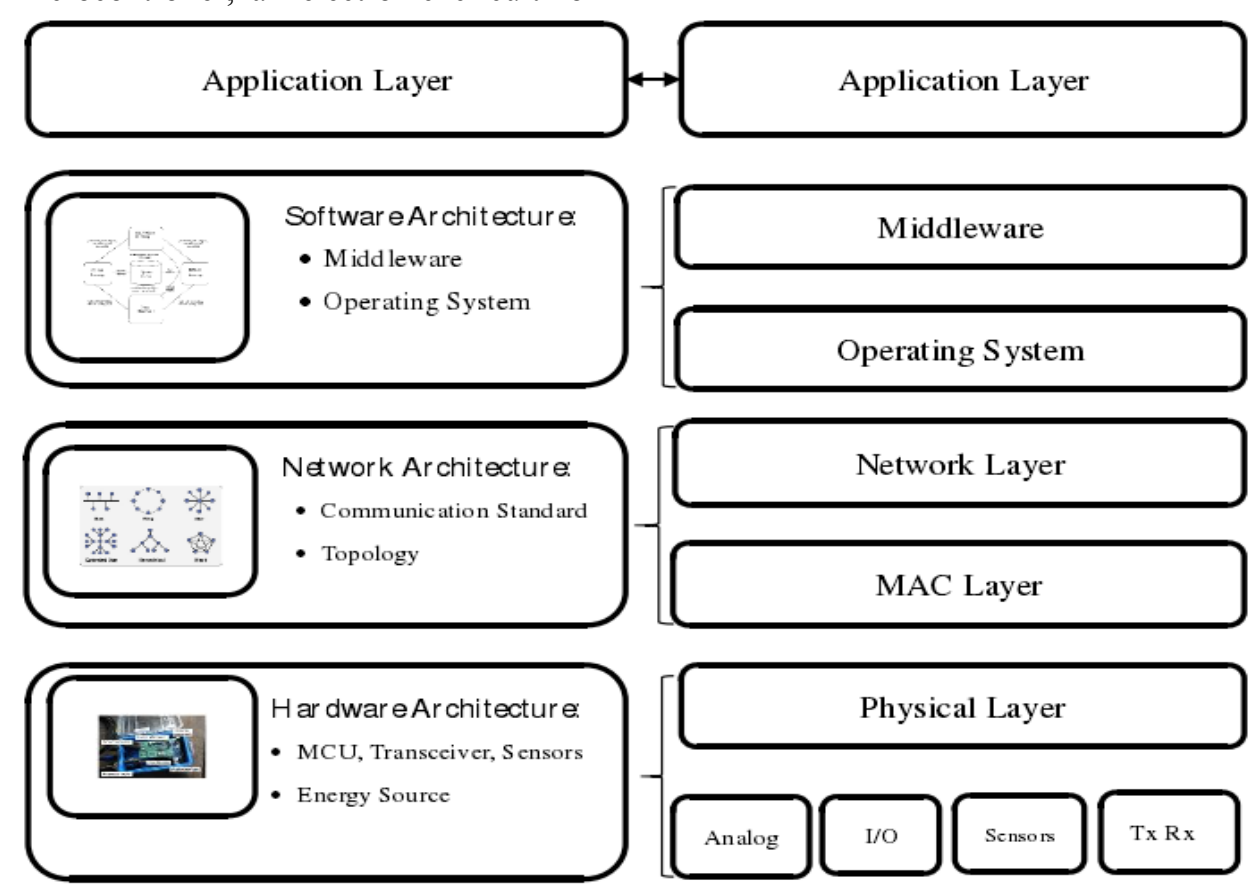

Figure 1. Three main architectures composing a WSN platform architecture 
jority of the WSN deployed nodes. In order to reduce design and development complexities, the sink node's hardware architecture is often designed very similar or identical to the source node with the exception of unlimited power available to sink nodes.

The network architecture of a WSN platform refers to the network topology and wireless communication standard technology deployed. The software architecture, on the other hand, refers to the middleware architecture and the operating system of the WSN [5-7]. The middleware resides between the application layer and the operating system residing on the sensor nodes.

The hardware architecture of a wireless sensor node is composed of four essential components: microcontroller unit $(\mathrm{MCU})$, transceiver ( $\mathrm{TxRx})$, power source, and sensor-elements as seen in figure 2. The MCU controls and coordinates the functions of the sensor node, directs the transceiver, and monitors the sensor elements. The transceiver is the wireless communication device that transmits and receives data packets using radio frequency (RF) channels. Each such sensor node has typically several parts: a radio transceiver with an internal antenna or connection to an external antenna, a microcontroller, an electronic circuit for interfacing with the sensors and an energy source, usually a battery or an embedded form of energy harvesting. The power source is the energy source for the sensor node, which can be battery operated, AC powered, or using some form of energy harvesting (e.g. solar power). Sensor-elements (SE) are the actual sensing components inside the sensor node that extract information from the environment, such as temperature, pressure, light, sound, etc.

\section{METHOD AND ALGORITHM}

Library air quality monitoring involved capturing sensor values of various parameters associated with library air quality by using different gas sensors. The system consists of a remote control center platform serving as a processing unit and an interface board for sensors and radio modules. We used three motes, and each one of them is nothing but a remote control center platform containing a sensor board with all sensors together with XBee modules; due to the DigiMesh protocol, all the motes act like homogeneous nodes. remote control center gateways along with XBee module form a base station or simply a gateway. The gateway acts as a base station that receives and transmits information to sensor nodes in a wireless sensor network. A sensor node is nothing but combination of remote control center, sensor board and XBee module. The sensor nodes are part of wireless network and directly talks with the gateway and with each other. A typical wireless mesh network using remote control center is shown in Figure 3 and the block diagram of the LAQ monitoring system deployed in this work is given in figure 4 .

This section discusses the architecture of sensor nodes and the gateway, the two main components of the wireless mesh network, which consist the LAQ monitoring system. Sensor nodes are nothing but a stack of several boards starting with remote control center platform as a main unit, a sensor board and a DigiMesh XBee radio module connected to the top of the remote control center, which makes the system look compact in size and very handy to use. Throughout this section, a detailed review of each of the components of the remote control center will be highlighted.

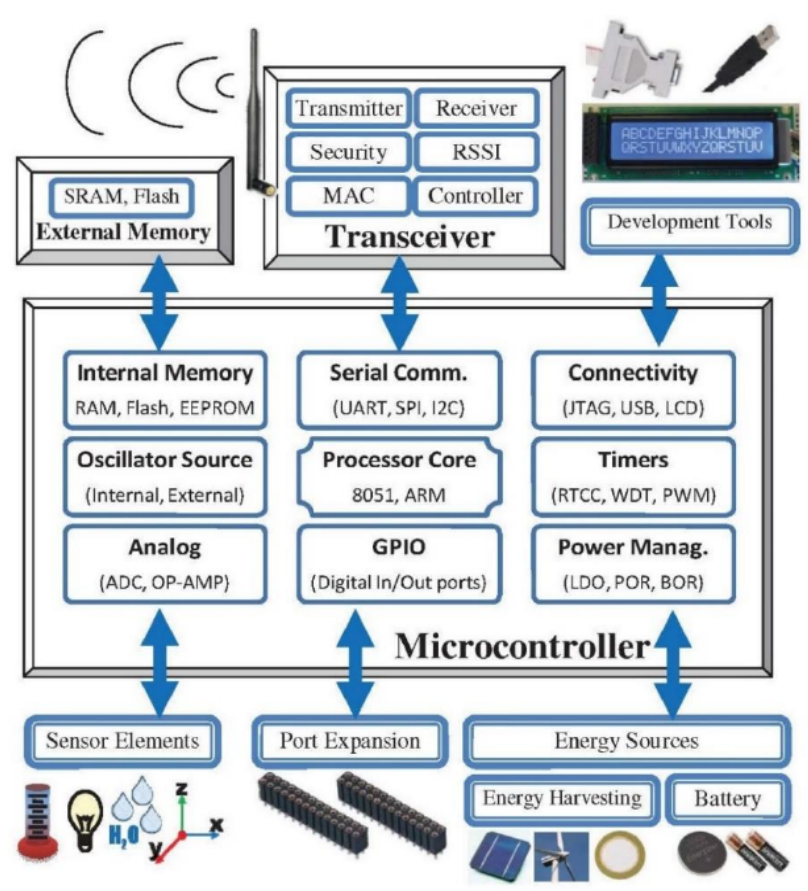

Figure 2. General hardware architecture of WSN platforms

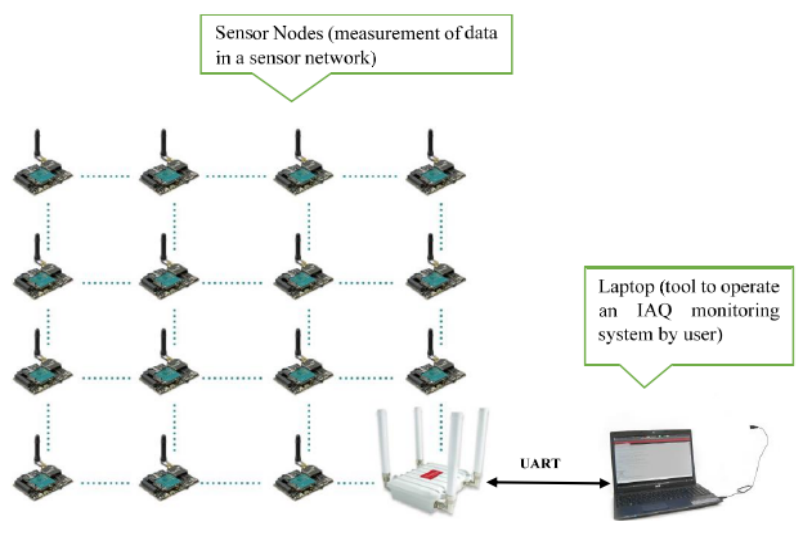

Figure 3. Magnetization as a function of applied field. Note how the caption is centered in the column

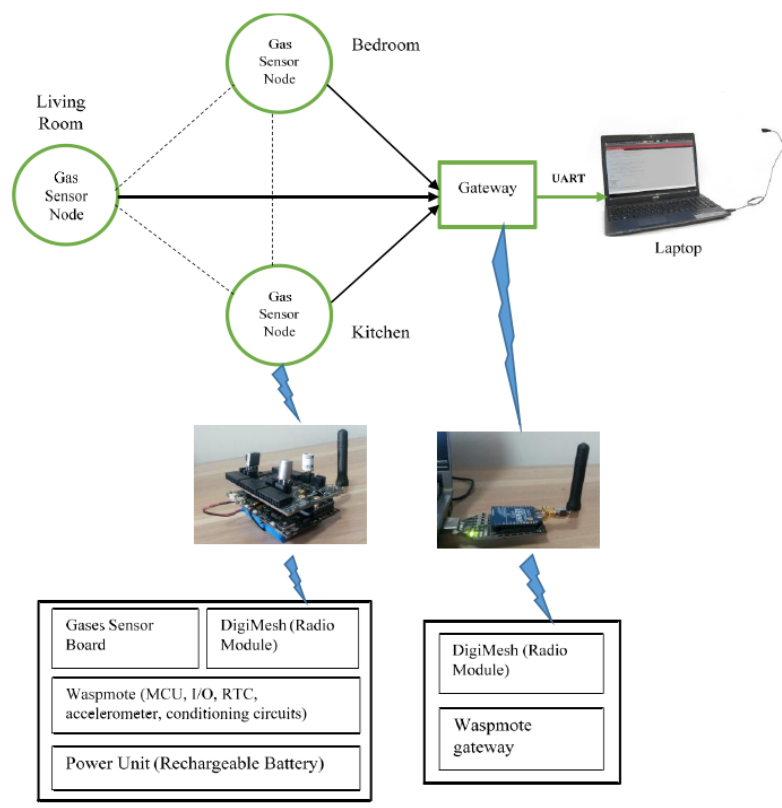

Figure 4. Block diagram of LAQ monitoring system 
The remote control center has a real time clock (RTC) module that can be programmed and controlled through the I2C bus. The real time clock module stores and automatically updates the time for remote control center and allows remote control center to be programmed to finish specific tasks including waking up after sleeping for a specific time or waking up every day at a specific time to perform corresponding tasks.

The core part of real time clock module is the alarm clocks that can be programmed in two specific formats including day/hour/minute/second and hour/minute/second. Alarm clocks give users total control when remote control center wakes up to capture sensor values and perform programmed actions and when remote control center changes into saving energy mode. In other words, alarms allow remote control center to be in deep sleep mode or hibernate mode for saving energy and make remote control center wake up just at the required moment.

The real time clock module is based on the hardware DS3231SN that has a frequency of $32.786 \mathrm{~Hz}$, which allows real time clock module to calculate time variations with high precision. As one of the accurate clocks, DS3231SN uses internal compensation mechanism and has a loss of just $2 \mathrm{ppm}$ which equals to variation of $0.16 \mathrm{~s}$ per day while most RTCs on market has a $1.7 \mathrm{~s}$ loss of accuracy per day.

Onboard sensors are divided into analog and digital sensors. Analog sensors require an analog-to-digital converter (ADC) on the MCU to convert variable voltage levels from the sensor into digital readings. Digital values can then be translated to meaningful sensor related units (e.g. Celsius for temperature) by using a lookup table in the internal Flash memory or by using a conversion equation. On the other hand, digital sensor elements are already equipped with an $\mathrm{ADC}$ and provide meaningful sensor readings.

Communication with a digital sensor element is typically done using a standard serial interface, such as DART, SPI, I2C, etc. Digital sensor elements that use a common shared type of serial communication bus, such as I2C, can scale in number easier than any other alternative due to the limited number of GPIOs on a given MCU. Therefore, providing a commonly shared serial bus to connect sensors is recommended for scalability.

There are more than 60 sensors available to connect to remote control center: $\mathrm{CO}, \mathrm{C} 02$, soil moisture, presence, humidity, temperature, vehicle detection, radiation, current, liquid, luminosity, etc. These sensors are connected to remote control center through different sensor boards. In this research, a gases sensors board is used together with remote control center to detect library environment parameters.

\section{EXPERIMENT RESULT}

Two places; a circulation desk and a reading room were chosen in the apartment of students at library of Peking University for testing purpose and sensor nodes were placed in those two rooms. The gateway and the monitor, which is a personal computer, are also placed in the reading room.

The gateway and sensor nodes and deployed in room $70 \_5$, block_5 of library of Peking University. Sensor nodes for testing were placed in two different places, but they were still close and no issues with the test range oc-

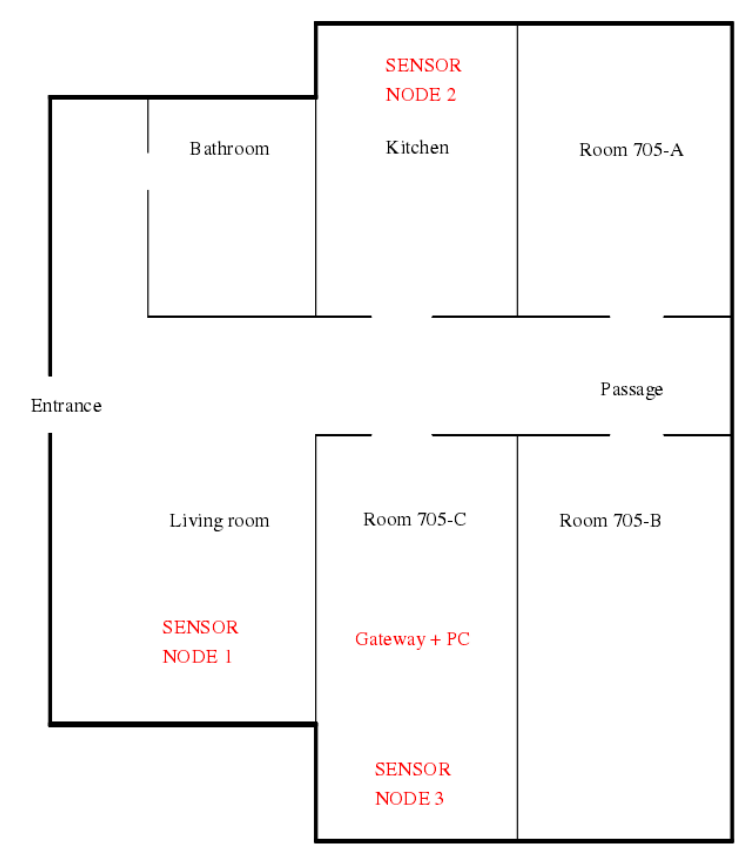

Figure 5. Experimental set up

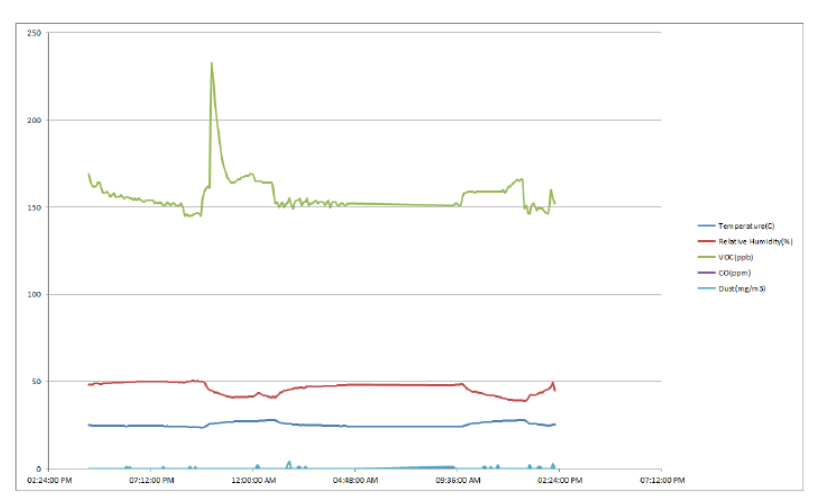

Figure 6. Experimental sensor value

curred. All nodes are in a mesh network; therefore they have the same behavior of talking to each other and sending packets to the gateway. The gateway transmits data to the computer for further processing. Experimental set up for the LAQ monitor is shown in figure 5.

The experimental sensor value is shown in figure 6. A Tree topology is a hierarchal type network that starts from the level-one root node, or parent node, and braches out into sub-levels, such that the root node is connected to one or more level-two sub-nodes, or children nodes. The middleware is the software layer located between the application layer and the operating system residing on the sensor node. The middleware's job is to assist the application layer in composing applications for the WSN platform while hiding details about the underlying operating system, network, and hardware level details. In addition, the middleware defines the software orientation of a given WSN platform.

\section{CONCLUSION}

A low cost LAQ monitoring system is developed to give clearer and more detailed view of library air quality and will be beneficial in many low cost applications. Also such system is in reach of all individuals irrespective of economical class. This system is extension for all availa- 


\section{SHORT PAPER}

\section{A WIRELESS SENSOR NETWORKS-BASED INTELLIGENT SYSTEM FOR LIBRARY AIR QUALITY MONITORING}

ble LAQ monitoring system, which are working on the principle of place-to-place gas detection i.e. without using wireless network to monitor gas detection over a larger area. In addition to that, this system provides some additional features like continuous data monitoring from more than one place, gas detection in hazardous areas, monitoring at different parts of the building at the same etc. than available LAQ monitoring systems.

The overall cost of this system is around 8 50\$ when using 2 sensor nodes and $200 \$$ extra for each additional sensor node. This is price is much cheaper as compared to existing systems in market and provides much more additional functions in terms of more sensors, more functionality for data processing and availability of data in public.

Sensor nodes can reconfigure remotely over a wireless mesh network and most of the time they are in sleep mode, which saves energy consumption as they wake to detect sensor data when needed. This process also reduces communication load as reduction in packet size.

\section{ACKNOWLEDGMENT}

\section{REFERENCES}

[1] Y. Lin, J. Yang, Z. Lv, et al., "A self-assessment stereo capture model applicable to the internet of things," Sensors, vol. 15, no. 8, pp. 20925-20944, August 2015. https://doi.org/10.3390/s150820925

[2] H. Jing, "Node deployment algorithm based on perception model of wireless sensor network," International Journal of Automation Technology, vol. 9, no. 3, pp. 210-215, April 2015. https://doi.org/10.20965/ijat.2015.p0210

[3] K. S. Chia, and X. Y. Yap, "A Portable PID Control Learning Tool by means of a Mobile Robot," International Journal of
Online Engineering, vol. 12, no.6, pp. 54-57, June 2016. https://doi.org/10.3991/ijoe.v12i06.5716

[4] H. Yang, et al., "Toward resilient security in wireless sensor networks," Proceedings of the 6th ACM international symposium on Mobile ad hoc networking and computing ACM,pp.34-45, 2015.

[5] D. Jiang, X. Ying, Y. Han, et al., "Collaborative Multi-hop Routing in Cognitive Wireless Networks," Wireless Personal Communications, vol. 86, no.2, pp. 901-923, January 2016. https://doi.org/10.1007/s11277-015-2961-6

[6] Z. Sun, Y. Zhang, Y. Nie, et al., "CASMOC: a novel complex alliance strategy with multi-objective optimization of coverage in wireless sensor networks," Wireless Networks, pp. 1-22, 2016. https://doi.org/10.1007/s11276-016-1213-3

[7] H. Jing, "Routing optimization algorithm based on nodes density and energy consumption of wireless sensor network," Journal of Computational Information Systems, vol. 11, no. 14, pp. 50475054 , July 2015.

\section{AUTHORS}

Zhang Xiao-guang is with Hebei North University, Hebei, China (404542905@qq.com).

Zhao Yijie is with Hebei North University, Hebei, China.

Zhao Wei is with Hebei North University, Hebei, China.

Xu Wei-wei is with Zhangjiakou Electronic Education, Hebei, China.

Ji Wen-chao is with Hebei North University, Hebei, China.

The paper is supported by 2013 Hebei North University surfaces university research project. Submitted 09 September 2016. Published as resubmitted by the authors 14 October 2016 . 